\title{
Effect of microwave-assisted extraction on bioactive compounds from industrial tomato waste and its antioxidant activity
}

\author{
Lasunon, P., Phonkerd, N., Tettawong, P. and *Sengkhamparn, N. \\ Faculty of Interdisciplinary Studies, Khon Kaen University, Nong Khai Campus, Nong Khai, 43000, \\ Thailand
}

\begin{abstract}
Article history:
Received: 19 September 2020

Received in revised form: 27

October 2020

Accepted: 17 December 2020

Available Online: 29 April

2021
\end{abstract}

Keywords:

Lycopene,

Phenolic,

Flavonoid,

Microwave-assisted

extraction,

Antioxidant activity,

Fuzzy assessment

DOI:

https://doi.org/10.26656/fr.2017.5(2).516

\section{Introduction}

Tomatoes are one of the healthy plants which contain high nutrients and bioactive compounds, for example, carotenoids and phenolic compounds (Fennema, 1996; Eh and Teoh, 2012; Sengkhamparn and Phonkerd, 2019; Sengkhamparn et al., 2019). They can be used in many products in the industry which creates huge waste. The tomato waste from each industry varies depending on the final product. The tomato waste from the tomato paste industry has been reported as containing seed, skin and also some pulp (Kaur et al., 2008). Many researchers have used them for bioactive compound extraction especially carotenoid and some report on the phenolic compound. Besides, they can be used as a functional ingredient for gelatin film (Jirukkakul and Sodtipinta, 2017) as well as for crisp products (Jirukkakul, 2017).

Microwave-assisted extraction (MAE) is a highefficiency technique for bioactive compounds from many plants due to the rapid heat generation. MAE can perform shorter extraction and a lower solvent level compared to conventional methods and can be further developed for industrial purposes (Mandal et al., 2007; Prakash Maran et al., 2013; Tongkham et al., 2017)
However, the extracted bioactive compound can be affected by MAE process. Even though, the increase of microwave power caused the increase of extract yield, the high power of microwave use for extraction can degrade some bioactive compounds (Chan et al., 2011). Moreover, the bioactive compound selectivity is dependent on the extracted solvent, therefore, fractionation is performed (Chan et al., 2011). However, the bioactive compounds in tomatoes contain both hydrophobic compounds namely lycopene, and also hydrophilic compounds namely phenolic and flavonoid, therefore, after extraction the fractionation should be performed. However, the effect of MAE use on lycopene and phenolic compounds as well as flavonoid from tomato waste powder have not been reported.

The fuzzy assessment method (FAM) is a mathematical technique that has been reported to be used to decide the fuzzy condition. The results can be generated as a fuzzy set by a membership function in the range of 0 to 1 and then all criteria were integrated into the overall performance index in which can be used for comparison and for determination or decision. FAM has been performed for assessment, such as for food product quality control (Perrot et al., 2006), for sensory evaluation in drinks from bread (Singh et al., 2012), dahi 
(Indian yoghurt) powder (Routray and Mishra, 2012), and rice-cracker (Lasunon and Sengkhamarn, 2015), and also for deciding the best condition for pectin extraction from dragon fruit peel (Tongkham et al., 2017).

Therefore, the effect of MAE use followed by fractionation on lycopene and phenolic compounds from industrial tomato waste has been studied. The antioxidant activity of each obtained extract was also determined. The fuzzy assessment method was performed to decide the best condition.

\section{Materials and methods}

\subsection{Materials}

The tomato waste from the tomato paste industry was composed of seed, pulp and skin in which bioactive compound was different in each part for example the phenolic compound has been rich in seeds while lycopene has been rich in skins. This tomato waste was dried at $60^{\circ} \mathrm{C}$ for $24 \mathrm{hrs}$. Then, it was ground, vacuumpacked and stored at $-18^{\circ} \mathrm{C}$ before further experimentation.

\subsection{Microwave-assisted extraction}

The microwave-assisted extraction was performed by adding $5 \mathrm{~g}$ of tomato waste powder to $100 \mathrm{~mL}$ of $95 \%$ Ethanol and microwaved at different powers of 180, 300 and $450 \mathrm{~W}$ and for 30,60 and $90 \mathrm{~s}$, respectively by using a $2450 \mathrm{MHz}$ household microwave oven. The temperature of suspension after microwave heated from each condition is shown in Table 1. After centrifuge, the supernatant was fractionated into hydrophobic and hydrophilic part by using hexane containing $0.1 \%$ BHT to preventing oxidation during fractionation. The hydrophobic fraction (hexane part) was added with sodium sulphate anhydrous in order to remove some of the water and then remove the hexane by rotary evaporator. The crude extract was adjusted to $5 \mathrm{~mL}$ by $\mathrm{n}$ -

hexane to control the volume of extracts. The hydrophilic fraction (ethanol part) was collected and concentrated by using a rotary evaporator and adjusted to $25 \mathrm{~mL}$ of ethanol. The crude extracted was stored at $18^{\circ}$ $\mathrm{C}$ for further analysis.

\subsection{Carotenoid content in the hydrophobic fraction}

The carotenoid contents in the hydrophobic fraction were determined using High Performance Liquid Chromatography HPLC (Shimadzu LC-20A, Kyoto, Japan) equipped with $\mathrm{C} 18$ reverse phase columns $(25 \mathrm{~cm}$ $\times 4.6 \mathrm{~mm}, 5 \mu \mathrm{m}$ id.) and photodiode array (Shimadzu SPD-M20A, Kyoto, Japan) at $450 \mathrm{~nm}$. The acetonitrile/ dichloromethane/methanol $(50: 40: 10 \mathrm{v} / \mathrm{v} / \mathrm{v})$ was used as mobile phase with a flow rate of $1 \mathrm{~mL} / \mathrm{min}$. The translycopene from tomato and beta-carotene were used as standard.

\subsection{Total phenolic compound and total flavonoid content in the hydrophilic fraction}

The total phenolic compound (TPC) and total flavonoid content (TFC) in the hydrophilic fraction were determined according to Sengkhamparn and Phonkerd (2019) and the result was compared to standard gallic acid and catechin, respectively.

\subsection{DPPH radical scavenging activity}

The antioxidant activity of hydrophobic and hydrophilic fraction was determined. The DPPH radical scavenging activity of the hydrophobic fraction was determined according to Erkan et al. (2008) by adjusted the polarity of extracted with ethyl acetate, while the hydrophilic fraction was determined according to Sengkhamparn and Phonkerd (2019). The results were expressed as a percentage of radical scavenging activity $(\%)$ as the following equation:

Radical scavenging activity $(\%)=\left(A_{\text {control }}-A_{\text {sample }}\right) \times 100 /$ A control

Table 1. The total phenolic compound and flavonoid content in the hydrophilic fraction

\begin{tabular}{|c|c|c|c|c|}
\hline \multicolumn{2}{|c|}{ Extraction condition } & \multirow{2}{*}{$\frac{\text { Temperature }\left({ }^{\circ} \mathrm{C}\right)}{34.67 \pm 1.00}$} & \multirow{2}{*}{$\begin{array}{c}\text { Total Phenolic Compound } \\
\text { (mg GAE/100 g DM) }\end{array}$} & \multirow{2}{*}{$\begin{array}{c}\text { Total Flavonoid Content } \\
(\mathrm{mg} \mathrm{CE} / 100 \mathrm{~g} \mathrm{DM})\end{array}$} \\
\hline \multirow{3}{*}{$180 \mathrm{~W}$} & $30 \mathrm{~s}$ & & & \\
\hline & $60 \mathrm{~s}$ & $40.27 \pm 0.55$ & $260.53 \pm 4.43^{\mathrm{ab}}$ & $6863.75 \pm 267.97^{\mathrm{f}}$ \\
\hline & $90 \mathrm{~s}$ & $47.00 \pm 0.61$ & $187.57 \pm 8.06^{\mathrm{c}}$ & $9832.52 \pm 174.27^{\mathrm{a}}$ \\
\hline \multirow{3}{*}{$300 \mathrm{~W}$} & $30 \mathrm{~s}$ & $40.57 \pm 0.59$ & $140.79 \pm 4.84^{\mathrm{d}}$ & $7801.63 \pm 401.95^{\mathrm{cd}}$ \\
\hline & $60 \mathrm{~s}$ & $51.33 \pm 0.38$ & $107.73 \pm 0.81^{\mathrm{d}}$ & $8567.25 \pm 57.42^{b}$ \\
\hline & $90 \mathrm{~s}$ & $61.93 \pm 0.40$ & $229.71 \pm 42.52^{\mathrm{b}}$ & $7849.53 \pm 296.72^{\text {bcd }}$ \\
\hline \multirow{3}{*}{$450 \mathrm{~W}$} & $30 \mathrm{~s}$ & $46.63 \pm 0.86$ & $280.10 \pm 10.68^{\mathrm{a}}$ & $6975.13 \pm 149.65^{\text {ef }}$ \\
\hline & $60 \mathrm{~s}$ & $63.4 \pm 0.00$ & $134.67 \pm 31.78^{\mathrm{d}}$ & $8516.74 \pm 261.05^{\mathrm{bc}}$ \\
\hline & $90 \mathrm{~s}$ & $73.97 \pm 0.78$ & $183.76 \pm 13.47^{\mathrm{c}}$ & $8475.11 \pm 692.62^{\mathrm{bc}}$ \\
\hline
\end{tabular}

Values are expressed as mean \pm standard deviation. Values with different superscript within the column are significantly different $(\mathrm{p}<0.05)$. 
Where $\mathrm{A}_{\text {sample }}$ was the absorbance of the mixture and $\mathrm{A}_{\text {control }}$ was the absorbance of the ethyl acetate and ethanol instead of crude extract for hydrophobic fraction and hydrophilic fraction, respectively.

\subsection{Statistical analysis}

The experimental design was done using a $3 \times 3$ (microwave power $\times$ extraction time) full factorial design. All experiments were done in triplicate and the treatment comparison was performed by Duncan's New Multiple Range Test at the significance level of $p<0.05$.

\subsection{Fuzzy assessment method}

In order to determine the most suitable conditions for bioactive compound extraction using the microwave technology, a fuzzy assessment method was calculated according to Lasunon (2016). The overall performance index under fuzzy theory was calculated by using 2 quality criteria which were the values for DPPH radical scavenging activity of hydrophobic and hydrophilic fraction with the same weight of 50. Briefly, the overall performance index was calculated by using a performance score in which the antioxidant activity was calculated to score from 0 to 10 by using the lowest and highest value for each of the criteria. The performance score was then converted to a fuzzy performance grade matrix in order to integrate the overall performance index with their relative weights (Tongkham et al., 2019).

\section{Results and discussion}

\subsection{Carotenoid content in the hydrophobic fractions}

Tomatoes are one of the richest in nutrients vegetables and can be industrially processed into many products. This research has used industrial tomato waste as a raw material for bioactive compound extraction. The microwave-assisted extraction (MAE) is an extraction technique that has been reported to be used for bioactive compound extraction. However, the conditions of MAE would be affected by the amount and activity of the extract. The bioactive compound in tomato waste powder was extracted by MAE using $95 \%$ ethanol in different conditions based on the temperature of the suspension in which the extraction temperature was not in excess of $77^{\circ} \mathrm{C}$ and then the extract was fractionated into 2 parts using hexane/BHT. The trans-lycopene and betacarotene contents in hydrophobic fraction (Hexane part) were determined by HPLC techniques.

From Figure 1, it was found that the MAE condition affected the carotenoid content. With the increase of microwave power levels from $180 \mathrm{~W}$ to $300 \mathrm{~W}$, the trans -lycopene, as well as beta-carotene content, were increased. This was probably due to the higher microwave power, the higher temperature of suspension which led to the higher extractability of bioactive compounds. When setting high extraction temperatures or high microwave power levels, and enhancement of the rupture of the cell material was increased which could enhance the solvent penetration into the plant material. However, with the increase of microwave power levels from $300 \mathrm{~W}$ to $450 \mathrm{~W}$, the amounts of trans-lycopene, as well as beta-carotene, were decreased. This was probably due to the degradation of trans-lycopene and betacarotene during the extraction. This result was in accordance with Chan et al. (2011) who states that the extraction at high power levels caused the degradation of bioactive compounds. Moreover, it may be due to the thermal degradation of lycopene at a high microwave power $(450 \mathrm{~W})$ when the temperature of suspension was in the range of $47-75^{\circ} \mathrm{C}$ (Table 1). Considering the effect of extraction time, it was found that most lycopene and beta-carotene were obtained from a longer extraction. This can be explained by the longer the heat generation, the greater solubility of bioactive compounds in the solvent. The temperature of suspension during the extraction affected the amounts of trans-lycopene and beta-carotene. Hackett et al. (2004) reported that at the temperature of $25-30^{\circ} \mathrm{C}$, the lycopene could be oxidized meanwhile the that $75-100^{\circ} \mathrm{C}$, the lycopene could be isomerized. However, in our study, the temperature of suspension was in the range of $35-75^{\circ} \mathrm{C}$. In this study, the results presented that the highest carotenoid content (5.74 mg lycopene/100 g tomato waste powder and 4.83 $\mathrm{mg}$ beta-carotene $/ 100 \mathrm{~g}$ tomato waste powder) was obtained from the extraction at a microwave power of $300 \mathrm{~W}$ for $90 \mathrm{~s}$. This result was lower than the research conducted by Ho et al. (2015) which reported that all trans lycopene could be extracted at high levels from tomato peel by using the microwave power of $400 \mathrm{~W}$ for $60 \mathrm{~s}$ and form this condition yielded the all-translycopene of $13.592 \mathrm{mg} / 100 \mathrm{~g}$ yield extract. However, the lycopene content in this study was higher than the research conducted by Kaur et al. (2008) in which lycopene $(1.98 \mathrm{mg} / 100 \mathrm{~g}$ tomato skin) was extracted from tomato processing waste skin at an extraction temperature of $50^{\circ} \mathrm{C}$ for 8 mins and using a Hexane/ Acetone/Ethanol $(2 / 1 / 1 \mathrm{v} / \mathrm{v} / \mathrm{v})$ as an extractant solvent and higher than the research conducted by Poojary and Passamonti (2015) in which lycopene (3.47-4.03 mg/100 g tomato pulp) was extracted from tomato pulp using an extraction temperature of $20^{\circ} \mathrm{C}$ for 40 mins. This was probably due to the extraction conditions such as solvent and also the raw material. The raw material that was used in this study contained seeds, pulp and also peel which was directly obtained from the tomato paste industry and were not separated into each type of waste. 
Moreover, ethanol was used as a solvent in this study which according to Calvo et al. (2007), ethanol is a more effective solvent to extract remaining lycopene in tomato peel at temperatures of 25 to $60^{\circ} \mathrm{C}$ than ethyl acetate. Moreover, ethanol is a non-toxic organic solvent and a good microwave absorber (Chan et al., 2011). However, the fractionation by hexane has been performed to increase the selectivity of the extraction.

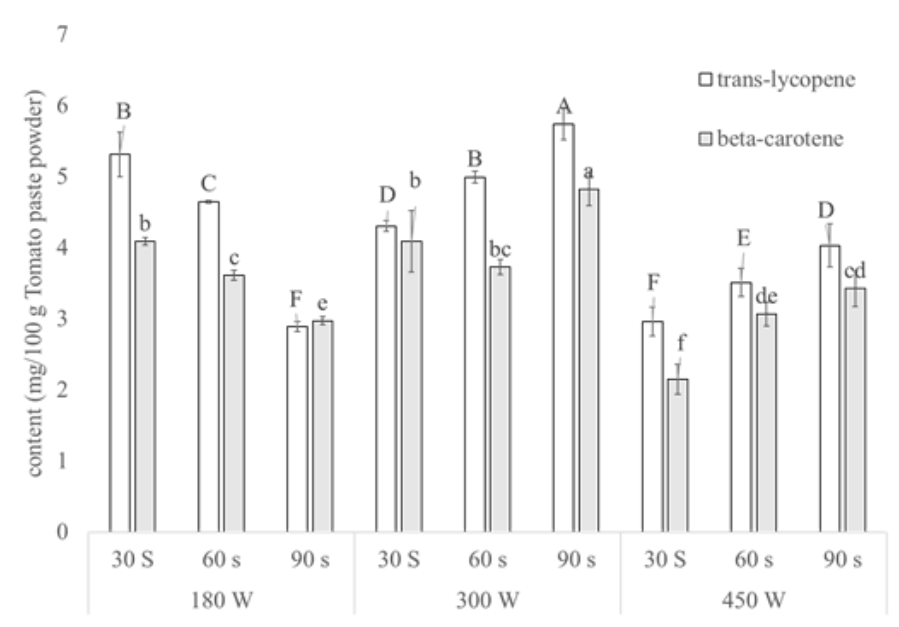

Figure 1. The carotenoid contents of the hydrophobic part. Bars with different uppercase letters and different lowercase letters are significantly different $(\mathrm{p}<0.05)$ among translycopene and beta-carotene respectively.

\subsection{Total phenolic and total flavonoid content in the hydrophilic fractions}

Despite lycopene, the phenolic compound can be also found in tomato, as reported by Vallverdú-Queralt et al. (2012). This bioactive compound has a hydrophilic property. Therefore, after fractionation, the total phenolic content (TPC) and total flavonoid content (TFC) of the alcoholic fraction were determined. The results are shown in Table 1.

The results showed that the microwave power affected the total phenolic content in which there was no significant difference of the TPC obtained from the microwave powers of 180 and $450 \mathrm{~W}$ but higher than that of $300 \mathrm{~W}$. The condition which gave the highest phenolic content was the microwave power of $450 \mathrm{~W}$ for $30 \mathrm{~s}$ in which the TPC was $280.10 \mathrm{mg}$ GAE/100 g DM. Additionally, the condition that gave the lowest TPC (107.73 mg GAE/100 g DM) was found at the microwave power of $300 \mathrm{~W}$ for $60 \mathrm{~s}$. This probably due to the thermal degradation of phenolic during extraction which the temperature in this condition was approximately $51.33^{\circ} \mathrm{C}$ which was optimum for polyphenol oxidase $\left(55^{\circ} \mathrm{C}\right)$ (Shahriar and Bahaaldin, 2013) and caused the degradation of phenolic compounds. However, the phenolic compounds could be extracted by MAE in the range of $107-280 \mathrm{mg}$ $\mathrm{GAE} / 100 \mathrm{~g}$ DM which was higher than in fresh tomatoes which were reported by Vallverdú-Queralt et al. (2012). Moreover, this result was lower than the research conducted by Li et al. (2012) which extracted phenolic compounds from tomato by MAE and reported TPC was in the range of 489.30-997.45 $\mathrm{mg}$ gallic acid equivalent/100 $\mathrm{g}$ dry weight.

For TFC content, the results exhibited that TFC was affected by the extraction time in which the longer extraction gave the higher TFC. This could be explained that by the greater of release bioactive compound to solvent during extraction. Moreover, it was found that the TFC was higher levels than TPC which was in agreement with Vallverdú-Queralt et al. (2012) who reported that flavanones and flavonols were the main phenolic compounds observed in each step of tomato paste processing. The best extraction condition giving the highest TFC (9832.52 $\mathrm{mg} \mathrm{CE} / 100 \mathrm{~g} \mathrm{DM}$ ) was the microwave power of $180 \mathrm{~W}$ for $90 \mathrm{~s}$ followed by the condition which performed at the microwave power of $300 \mathrm{~W}$ for $60 \mathrm{~s}$. The latter condition was found to yield the lowest phenolic contents. This was probably due to the extraction temperature of this condition was about almost $50^{\circ} \mathrm{C}$ which was close to the temperature at which the activity of polyphenol oxidase in tomato was the highest $55^{\circ} \mathrm{C}$ (Shahriar and Bahaaldin, 2013), hence the phenolic compound was degraded higher than others.

\subsection{Antioxidant activity}

The quality of the extracts has to be considered as well as the quantity of the extracts to choose the best extraction condition. Moreover, trans-lycopene and betacarotene have been reported as having a high efficacy for scavenging free radical. Besides, the phenolic and flavonoid compound also present as antioxidant compound. Therefore, the DPPH radical scavenging activity of hydrophobic fraction and also hydrophilic fraction were determined. The results are shown in Figure 2.

The DPPH radical scavenging activity of hydrophobic fraction has been affected by the microwave power and extraction time. The results exhibited that the high microwave power levels decreased the radical scavenging of the extracts, while the longer period of extraction increased the radical scavenging of the extracts. Furthermore, the MAE condition which gained the highest radical scavenging was the microwave power levels of $180 \mathrm{~W}$ and for $90 \mathrm{~s}$. However, this condition was not found to contain the highest amount of hydrophobic bioactive compound. This result indicated that the quantity of bioactive compound could not be represented the quality of extracts. The antioxidant activity of the extracted could be affected by many factors. Yi et al. (2009) reported 
that the trans- lycopene content, the ratio of lycopene isomer as well as other carotenoids or other bioactive compounds affected its antioxidant activity.

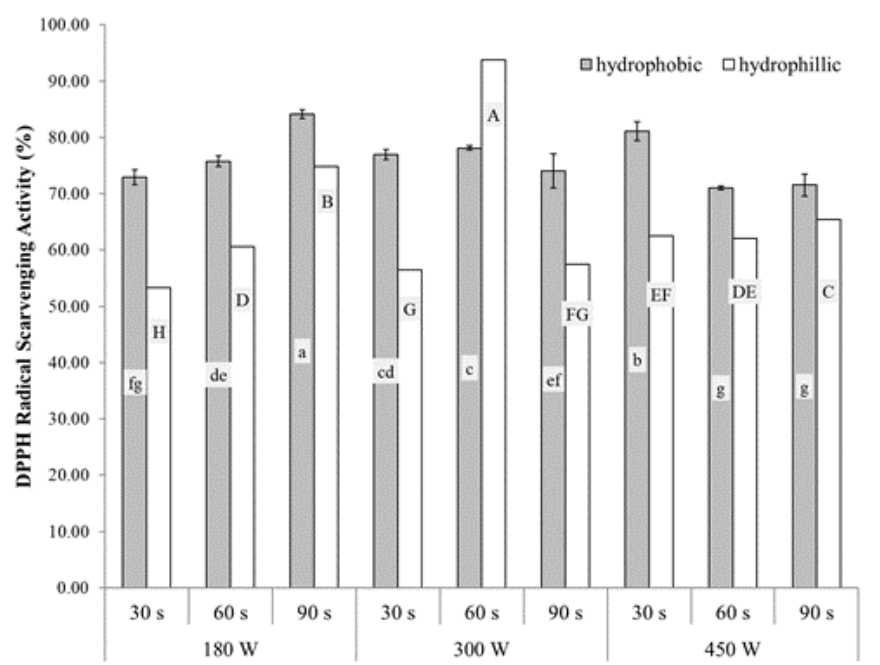

Figure 2. The DPPH radical scavenging activity of hydrophobic and hydrophilic fractions. Bars with different uppercase letters and different lowercase letters are significantly different $(\mathrm{p}<0.05)$ among the hydrophilic and the hydrophobic parts respectively.

On the other hand, the antioxidant activity of the hydrophilic fraction was also determined, and the results showed that the increased microwave power from 180 to $300 \mathrm{~W}$ increased the radical scavenging activity. This was probably due to the high amounts of bioactive compounds. In contrast, at the microwave power of 300 $\mathrm{W}$, the longer extraction gave the lower antioxidant activity. The high microwave power affected the structure of bioactive compounds as well as the amounts of bioactive compounds. The extract that used an extraction time of $60 \mathrm{~s}$ gave higher antioxidant activity than $90 \mathrm{~s}$. This can be explained by the thermal degradation during longer extraction. This result suggested that the extraction conditions would affect its antioxidant activity. The MAE condition giving the highest activity was the microwave power of $300 \mathrm{~W}$ for 60 s. However, this condition yielded low phenolic contents and considering the overall antioxidant activity together with the bioactive compound content of hydrophilic fractions, the correlation analysis found that the overall antioxidant activity was related to the flavonoid content with the correlation coefficient was 0.63 .

Interestingly, the MAE condition, which showed the highest antioxidant activity of hydrophobic fraction, gave a low content of carotenoid but gave the highest content of flavonoid in the hydrophilic fraction which the flavonoid content may have affected the hydrophobic fraction even though the fractionation step has been performed but the flavonoid may distribute to the hydrophobic fraction. Moreover, the highest antioxidant activity of hydrophilic fraction gave a high content of carotenoid as well. This could be a point that to study the extraction process in which the material contained high different bioactive compound, the composition of all bioactive compound and also structure of bioactive compound should be considered to decide the best extraction process which gave the maximum properties. Moreover, the method to determine its properties also be an imported criterion to consider. Li et al. (2012) reported that the optimal MAE for phenolic extraction from Tomato with maximum antioxidant properties was dependent on the antioxidant assay. They suggested that the best MAE condition for ferric reducing antioxidant power assay was the extraction at $96.5^{\circ} \mathrm{C}$ for $2.06 \mathrm{~min}$ using $66.2 \%$ ethanol, while the best condition for Oxygen radical absorption capacity assay was the extraction at $96.5^{\circ} \mathrm{C}$ for 1.66 min using $61.1 \%$ ethanol. To identify the best extraction condition for the high antioxidant activity, the fuzzy assessment method was applied and would be discussed in the next section.

\subsection{Fuzzy assessment method}

From the antioxidant activity of the extracts, it could be indicated that for bioactive compound extraction from tomato waste powder by the microwave technique, the best MAE condition depended on the bioactive compound. For hydrophobic compound, trans-lycopene and beta-carotene, the best MAE condition would perform at microwave power levels of $180 \mathrm{~W}$ for $90 \mathrm{~s}$ in which the extracts would show the highest quality (radical scavenging activity). In the meanwhile, the best MAE condition which gives the extracted with the highest activity was the extraction at microwave power of $300 \mathrm{~W}$ and time of $60 \mathrm{~s}$ for the hydrophilic compound. However, in the case of the extraction of all bioactive compound in the tomato wasted powder, the fuzzy assessment method would be applied in order to defined the best MAE condition.

In this work, the best condition for all bioactive compound extraction in tomato waste powder evaluated by fuzzy assessment method in which high antioxidant activity of both hydrophilic and hydrophobic fractions. The weight of the criteria was 50:50 for antioxidant activity of hydrophobic fraction and hydrophilic fraction, respectively. Moreover, the lower bound and upper bound for the criteria was 0 and $100 \%$, respectively. This value was used to calculate the overall performance score which was calculated by SciLab program (Lasunon, 2016) and the maximum and the minimum score was 10 and 0 , respectively. The results (Table 2) exhibited that the condition of $300 \mathrm{~W}$ for $60 \mathrm{~s}$ gave the highest overall performance index (8.601) and higher than that of $180 \mathrm{~W}$ for $90 \mathrm{~s}$ with the overall performance 
index was 7.857. This condition gave the highest antioxidant activity for hydrophobic fraction and hydrophilic part, respectively.

Table 2. The overall performance index from each MAE condition

\begin{tabular}{ccc}
\hline \multicolumn{2}{c}{ Extraction condition } & Overall performance index \\
\hline \multirow{3}{*}{$180 \mathrm{~W}$} & $30 \mathrm{~s}$ & 6.205 \\
& $60 \mathrm{~s}$ & 6.962 \\
& $90 \mathrm{~s}$ & 7.857 \\
\hline \multirow{3}{*}{$300 \mathrm{~W}$} & $30 \mathrm{~s}$ & 6.694 \\
& $60 \mathrm{~s}$ & 8.601 \\
& $90 \mathrm{~s}$ & 6.666 \\
\hline \multirow{3}{*}{$450 \mathrm{~W}$} & $30 \mathrm{~s}$ & 7.077 \\
& $60 \mathrm{~s}$ & 6.711 \\
& $90 \mathrm{~s}$ & 6.996 \\
\hline
\end{tabular}

\section{Conclusion}

The tomato waste powder from the tomato paste industry has been used for bioactive compound extraction namely trans-lycopene, beta-carotene as well as phenolic and flavonoid compounds. In this study, the MAE has been performed in various conditions in order to extract bioactive compounds by controlling the temperature, not excess $77^{\circ} \mathrm{C}$. After extraction, the crude extract was fractionated into a hydrophobic and hydrophilic fraction to improve the selectivity of the extract. It was found that the higher the microwave power, the higher the temperature of extraction condition enhanced the solubility of bioactive compounds, however, the increase in microwave power may degrade the purpose compounds which turn on the lower activity of the compound. Moreover, the longer the period of extraction, the larger the amount of extracted bioactive compounds, however, with the increase of microwave power and even though extraction time could degrade the bioactive compound. Considering the antioxidant, the exhaust of extraction conditions decreased its antioxidant activity. Moreover, the MAE condition which yielded the high bioactive compound would exhibit lower antioxidant activity. The best condition for a bioactive compound would depend on the type of bioactive compound. The overall performance index has been shown that the extraction condition of $300 \mathrm{~W}$ for $60 \mathrm{~s}$ was the best condition in which gave the high quality for the hydrophobic and the hydrophilic fraction.

\section{Conflict of interest}

The authors declare no conflict of interest.

\section{Acknowledgments}

The researchers gratefully thank the Research and Technology Transfer Affairs for their financial support
(Code 61003002).

\section{References}

Calvo, M.M., Dado, D. and Santa-Maria, G. (2007). Influence of extraction with ethanol or ethyl acetate on the yield of lycopene, $\beta$-carotene, phytoene, and phytofluene from tomato peel powder. Europe Food Research and Technology, 224, 567-571. https:// doi.org/10.1007/s00217-006-0335-8

Chan, R.Y., Ngoh, G.C. and Kung, F.W.L. (2011). Microwave-assisted extractions of active ingredients from plants. Journal of Chromatography A, 1218 (37), 6213- 6225. https://doi.org/10.1016/ j.chroma.2011.07.040

Eh, A.L.S. and Teoh, S.G. (2012). Novel modified ultrasonication technique for the extraction of lycopene from tomatoes. Ultrasonics Sonochemistry, 19(1), 151-159. https://doi.org/10.1016/ j.ultsonch.2011.05.019

Erkan, N., Ayranci, G. and Ayranci, E. (2008). Antioxidant activities of rosemary (Rosmarinus Officinalis L.) extract, blackseed (Nigella sativa L.) essential oil, carnosic acid, rosmarinic acid and sesamol. Food Chemistry, 110(1), 76-82. https:// doi.org/10.1016/j.foodchem.2008.01.058

Fennema, O.R. (1996). Food Chemistry. New York: Marcel Dekker Inc.

Hackett, M.M., Lee, J.H., Francis, D. and Schwartz, S.J. (2004). Thermal stability and isomerisation of lycopene in tomato oleoresins from different varieties. Journal of Food Science, 69(7), 536-541. https://doi.org/10.1111/j.1365-2621.2004.tb13647.x

Ho, K.K.H.Y., Ferruzzi, M.G., Liceaga, A.M. and San Martín-González, M.F. (2015). Microwave-assisted extraction of lycopene in tomato peels: Effect of extraction conditions on all-trans and cis-isomer yields. LWT-Food Science Technology, 62(1), 160168. https://doi.org/10.1016/j.lwt.2014.12.061

Jirukkakul, N. (2017). The Production and Development of Tomato Crisp from Tomato Pomace. Asia Pacific Journal of Science and Technology, 22, 120-130. https://doi.org/10.14456/apst.2017.12

Jirukkakul, N. and Sodtipinta, J. (2017). Effects of the tomato pomace oil extract on the physical and antioxidant properties of gelatin films. International Journal of Food Engineering, 3, 1-5. https:// doi.org/10.18178/ijfe.3.1.1-5

Kaur, D., Wani, A.A., Oberoi, D.P.S. and Sogi, D.S. (2008). Effect of extraction conditions on lycopene extractions from tomato processing waste skin using response surface methodology. Food Chemistry, 108 (2), 711-718. https://doi.org/10.1016/ 
j.foodchem.2007.11.002

Lasunon, P. (2016). Scilab software package for the fuzzy analytical method (FAM). Far East Journal of Mathematical Science, 100(2), 209-225. https:// doi.org/10.17654/MS100020209

Lasunon, P. and Sengkhamparn, N. (2016). Fuzzy analytical modeling for sensory evaluation of water meal (Wolffia arrhiza (L.) Wimm.) - Rice cracker. KKU Engineering Journal, 43(S2), 291-293

Li, H., Deng, Z., Wu, T., Liu, R., Loewen, S. and Tsao, R. (2012). Microwave-assisted extraction of phenolics with maximal antioxidant activities in tomatoes. Food Chemistry, 130(4), 928-936. https:// doi.org/10.1016/j.foodchem.2011.08.019

Perrot, N., Ioannou, I., Allais, I., Curt, C., Hossenlopp, J. and Trystram, G. (2006). Fuzzy concepts applied to food product quality control: A review. Fuzzy Set Systems, 157(9), 1145-1154. https://doi.org/10.1016/ j.fss.2005.12.013

Poojary, M.M. and Passamonti, P. (2015). Optimization of extraction of high purity all-trans-lycopene from tomato pulp waste. Food Chemistry, 188, 84-91. https://doi.org/10.1016/j.foodchem.2015.04.133

Prakash Maran, J., Sivakumar, V., Thirugnanasambandham, K. and Sridhar, R. (2013). Optimization of Microwave assisted extraction of pectin from orange peel. Carbohydrate Polymer, 97 (2), 703-709. https://doi.org/10.1016/ j.carbpol.2013.05.052

Routray, W. and Mishra, H.N. (2012). Sensory evaluation of different drinks formulated from dahi (Indian yogurt) powder using fuzzy logic. Journal of Food Process Preservation, 36(1), 1-10. https:// doi.org/10.1111/j.1745-4549.2011.00545.x

Shahriar, S. and Bahaaldin, R. (2013). Optimum Temperature and Thermal Stability of Crude Polyphenol Oxidase In Green Small Cherry Tomato (Solanum lycopersycum). International Research Journal of Applied and Basic Sciences, 4, 33063311.

Sengkhamparn, N. and Phonkerd N. (2019). Phenolic Compound Extraction from Industrial Tomato Waste by Ultrasound-Assisted Extraction. IOP Conference Series: Materials Science and Engineering, 639, 012040 . https://doi.org/10.1088/1757$899 X / 639 / 1 / 012040$

Sengkhamparn, N., Lasunon, P. and Tettawong, P. (2019). Effect of Ultrasound Assisted Extraction and Acid Type Extractant on Pectin from Industrial Tomato Waste. Chiang Mai University Journal of Natural Science. (Special Issue on Food and Applied Bioscience to Innovation and Technology), 18, 214-
225. https://doi.org/10.12982/CMUJNS.2019.0016

Singh, K.P., Mishra A. and Mishra H.N. (2012). Fuzzy analysis of sensory attributes of bread prepared from millet-based composite flours. LWT - Food Science and Technology, 48(2), 276-282. https:// doi.org/10.1016/j.lwt.2012.03.026

Tongkham, N., Juntasalay, B., Lasunon, P. and Sengkhamparn, N. (2017). Dragon Fruit Peel Pectin: Microwave-Assisted Extraction and Fuzzy Assessment Method. Agricultural Natural Resource, 51(4), 262-267. https://doi.org/10.1016/ j.anres.2017.04.004

Vallverdú-Queralt, A., Medina-Remón, A., CasalsRibes, I., Andres-Lacueva, C., Waterhouse, A.L. and Lamuela-Raventos, R.M. (2012). Effect of tomato industrial processing on phenolic profile and hydrophilic antioxidant capacity. LWT - Food Science and Technology, 47(1), 154-160. https:// doi.org/10.1016/j.lwt.2011.12.020

Yi, C., Shi, J., Xue, S.J., Jiang, Y. and Li, D. (2009). Effects of supercritical fluid extraction parameters on lycopene yield and antioxidant activity. Food Chemistry, 113(4), 1088-1094. https:// doi.org/10.1016/j.foodchem.2008.08.083 